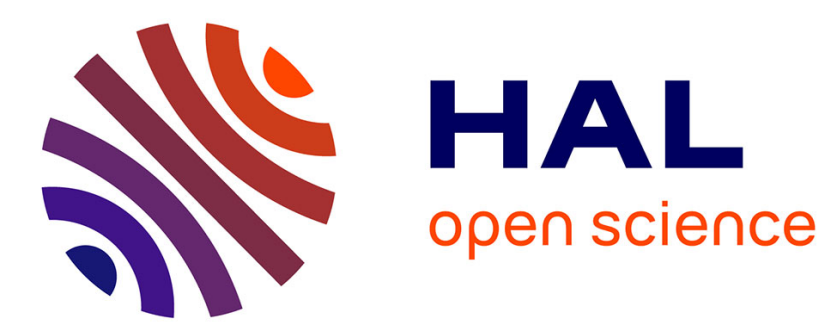

\title{
Les dîners de la Plume
}

Julien Schuh

\section{- To cite this version:}

Julien Schuh. Les dîners de la Plume. Romantisme: la revue du dix-neuvième siècle, 2007, 137, pp.79-101. 10.3917/rom.137.0079 . hal-00983956

\section{HAL Id: hal-00983956 https://hal.science/hal-00983956}

Submitted on 26 Apr 2014

HAL is a multi-disciplinary open access archive for the deposit and dissemination of scientific research documents, whether they are published or not. The documents may come from teaching and research institutions in France or abroad, or from public or private research centers.
L'archive ouverte pluridisciplinaire HAL, est destinée au dépôt et à la diffusion de documents scientifiques de niveau recherche, publiés ou non, émanant des établissements d'enseignement et de recherche français ou étrangers, des laboratoires publics ou privés. 


\section{Julien Schuh}

« Les dîners de la Plume : la vitrine d'un autre champ littéraire »

Les dîners de La Plume sont des casse-croûte où une cinquantaine de jeunes gens réunis se paient, pour cent sous, la tête d'un président chaque fois renouvelé.

Jules Renard, Journal, 19 septembre 1899.

En novembre 1892, Gabriel Randon (futur Jehan-Rictus) imagine dans le Mercure de France un épisode rocambolesque de la vie du critique Francisque Sarcey, célèbre pour ses opinions bourgeoises ridiculisées par les revues symbolistes ${ }^{1}$. Trois mois durant, Sarcey aurait souffert de ce mal décrit alors dans plusieurs ouvrages médicaux, le «génie », forme de névrose contagieuse poussant ses victimes aux pires excentricités. Le récit de cette crise, Trois mois de génie par un Homme de Bon Sens, contient un passage sur les Dîners de la Plume, qui ont débuté en mars de la même année :

8 mai. - J'ai été présenté à quelques jeunes gens pâles qui m'ont admis à présider un dîner appelé le « Dîner de la Plume ». Figurez-vous que c'était un dîner où l'on ne mange rien du tout. Les plats sont fictifs, et l'on somme de remporter les restes des domestiques supposés réels qui soi-disant circulent autour de vous. Ça, c'est très commode pour manger ce qu'on veut : il y en a qui préfèrent du perdreau, les autres des pâtés succulents, les autres des pâtisseries rares ; bien entendu, tout cela imaginaire ; moi, je ne savais pas qu'on pouvait se nourrir comme ça quand on était génial. Enfin! J'avais apporté un peu de saucisson que j'ai dévoré en cachette ${ }^{2}$.

Les banquets de la Plume étaient-ils donc si frugaux ? Les souvenirs et comptes rendus de l'époque permettent d'en douter; Randon ne fait sans doute ici que développer l'image des «nourritures spirituelles »: les jeunes poètes symbolistes se réunissent pour goûter des mets tout cérébraux. Mais ce passage montre que ces banquets, institués depuis quelques mois à peine, font déjà partie des rites fondamentaux de la bohême littéraire, puisque Sarcey génial s'oblige à y participer pour tenir son nouveau rôle de poète maudit. Ceci ne fait que confirmer l'importance de leur fonction symbolique, à peine esquissée ici : ces événements sont des rituels de légitimation, qui attestent le rôle d'une personnalité dans le panthéon des auteurs admirés par la jeunesse.

\footnotetext{
${ }^{1}$ Le même Sarcey se voit ainsi attribuer une parodie de compte rendu d'une soirée de la Plume: Francisque Sarcey [F.-A. Cazals ?], «Une Soirée au Quartier-Latin », La Plume, n 62, 15 novembre 1891, p. 396-401.

${ }^{2}$ Gabriel Randon, «Sarcey génial, conte invraisemblable », Mercure de France, n ${ }^{\circ}$ 35, novembre 1892, p. 231.
} 
Cette double fonction de reconnaissance d'un maître et de cohésion du groupe des littérateurs est à l'origine du projet des Dîners de la Plume. La revue organisait déjà des Soirées artistiques bien documentées et étudiées ${ }^{3}$; mais beaucoup de commentateurs et même de participants tendent à confondre Dîners ou Banquets (le terme est interchangeable dans les comptes rendus de ces événements) et Soirées ${ }^{4}$. Les Dîners, qui débutent en mars 1892 sous l'impulsion de Léon Deschamps, directeur de la Plume, ne sont au départ que de simples réunions amicales, comme l'explique le texte de l'invitation illustrée du premier banquet: «Dans le but de resserrer les relations amicales existant déjà entre un certain nombre d'entre nous, nous avons décidé de créer un dîner mensuel sous le titre : Dîner de LA PLUME ${ }^{5}$. » [Figure 1] Les fameux Dîners bimensuels chez Magny qui débutèrent en 1862 et réunirent la fine fleur de la littérature de l'époque (Sainte-Beuve, les frères Goncourt, Flaubert...) servirent sans doute de modèle à Deschamps ${ }^{6}$.

Dans la «Postface à l'année 1892 de "La Plume" », ce dernier revient cependant sur son initiative afin d'en préciser la portée : il s'agit de créer une manifestation sociale pour mettre fin à l'hostilité qu'il constate dans les milieux littéraires. La fonction des banquets s'inscrit dans une action globale destinée à permettre «l'avènement des nouvelles générations » par la propagande (la Revue), l'essai verbal des œuvres (les Soirées), l'anthologie (la Bibliothèque de la Plume), le culte des maîtres (les souscriptions pour divers monuments); reste à assurer le lien entre les générations littéraires et la paix au sein du groupe des gens de Lettres :

Supprimer l'acrimonie et la violence injurieuse dans les controverses littéraires et artistiques; opérer un changement total dans les relations entre maîtres et nouveaux venus ; remplacer envers les aînés la blague par le respect affectueux, cela en les faisant mieux connaître ; enfin provoquer une sorte de fusion des anciens et des plus jeunes, permettant à ces derniers l'accès des salles de rédaction et des librairies (Banquets de La Plume ${ }^{7}$ ).

Le début des années 1890 avait en effet été marqué par de violentes attaques ad hominem à travers la presse. L'Enquête sur l'évolution littéraire de Jules Huret est ainsi un triste constat sur l'unité du mouvement littéraire symboliste : René Ghil, Adrien Remacle, Charles Vignier, Gustave Kahn se complaisent dans la destruction des réputations de leurs anciens camarades.

\footnotetext{
${ }^{3}$ Voir Guy de Grosbois, «Il y a cent ans, les agapes de La Plume », La Revues des revues, ${ }^{\circ}$ 8, Association Ent'revues, hiver 1989, p. 21-24.

${ }^{4}$ Dîners et Soirées ont certes partie liée : si les Dîners n'ont lieu que tous les mois, ils sont toujours suivis d'une soirée «musicale et littéraire ».

${ }^{5}$ La Plume, $\mathrm{n}^{\circ} 69,1^{\mathrm{er}}$ mars 1892, p. 128.

${ }^{6}$ Voir Robert Baldick, Les Dîners Magny, Denoël, 1972.

${ }^{7}$ Léon Deschamps, « Postface à l'année 1892 de "La Plume” », La Plume, n 88, 15 décembre 1892, p. 536.
} 
Les interviews, les manifestes, les pamphlets prennent des allures de règlements de comptes, au point que Saint-Pol-Roux appelle à une «trêve» dans le Mercure de France d'octobre $1891^{8}$. Cette trêve, Deschamps tente de la produire par l'institution de ses Dîners. Lors de la première série des banquets de la Plume, treize artistes de la génération précédente viennent à tour de rôle présider l'une de ces réunions qui rassemblent un peu plus d'une centaine de convives issus de la «Jeunesse littéraire ». Le succès est rapide, comme le note le Figaro du 12 mai 1893: «la rédaction de la Plume organisa des banquets mensuels qui furent incontestablement une de ses principales causes de succès. Les célébrités du monde des lettres vinrent y occuper le fauteuil présidentiel. Ce furent tour à tour MM. Zola, Coppée, Vacquerie, Leconte de Lisle, Scholl, Mallarmé, Verlaine ; hier soir, le rédacteur en chef du Figaro, M. Magnard. N'est-ce pas une preuve d'esprit de la part de ces “chers maîtres"' que d'être venus s'asseoir parmi des jeunes qui les discutent parfois avec si peu de ménagements ${ }^{9}$ ! »

\section{Le banquet du Pèlerin passionné : un modèle du genre.}

Le choix de remettre en honneur la coutume des banquets littéraires est alors relativement neuf; Fontainas rappelle que le banquet commémorant la sortie du Pèlerin passionné de Moréas en février 1891 était alors une forme de nouveauté ${ }^{10}$ : «Les banquets littéraires n'étaient point encore d'un usage quotidien ; on n'en organisait ni chaque semaine, ni même chaque mois ; tout au plus, on en comptait un tous les deux ans ${ }^{11}$. » Ce banquet peut servir de modèle pour étudier les fonctions et les formes de ces réunions qui deviennent rapidement très populaires.

Sa première fonction est de cohésion. Après avoir posé l'origine rêvée du mouvement en plaçant ce banquet sous l'égide de Baudelaire, Verlaine et Mallarmé, les invités multiplient toasts et lectures de poèmes, dans l'espace d'un banquet qui est autant réel qu'idéal, celui du groupe littéraire symboliste, espace dans lequel se scelle «l'amitié des deux générations par la célébration d'un idéal commun, le culte de la poésie ${ }^{12} »$.

Mais sa fonction principale est de représentation. Le compte rendu du banquet de Remy de Gourmont dans le Mercure de France du mois de mars présente la « liste de Table des personnes présentes ${ }^{13}$ », où figurent entre autres Octave Mirbeau, Édouard Schuré, Pierre

\footnotetext{
${ }^{8}$ Saint-Pol-Roux, « La Gent irritable : La Trêve », Mercure de France, n ${ }^{22}$, octobre 1891, p. 194.

${ }^{9}$ Roland de Marès, « Les jeunes revues », Le Figaro, 12 mai 1893.

${ }^{10}$ Sur ce banquet et ses conséquences, voir Robert Jouanny, Moréas, écrivain français, Lettres modernes, 1969.

${ }^{11}$ André Fontainas, Mes Souvenirs du Symbolisme (1928), Éditions Labor, coll. Archives du Futur, Bruxelles, 1991, p. 76.

${ }^{12}$ Idem, p. 77.

${ }^{13}$ Remy de Gourmont, «Le Banquet du 2 février », Mercure de France, n 15, mars 1891, p. 190.
} 
Quillard, Charles Morice, Paul Gauguin, Alfred Vallette, Félicien Rops et bien d'autres, tous théoriciens, poètes ou peintres du mouvement. Cet étalage constant des listes des participants aux banquets (La Plume publie ainsi à la fin de chaque numéro la liste des présents à ses « soirées littéraires ») participe à la concrétisation d'un groupe littéraire, qui ne vit que des preuves qu'il donne de sa réalité. D'où l'importance de la présence de la presse, qui s’invite pour la première fois en masse à ses manifestations pour en rendre compte à ses lecteurs. Barrès, l'un des organisateurs du banquet du Pèlerin passionné, raconte au savant Byvanck de passage à Paris en 1891 l'arrivée des journalistes et des curieux dans la salle ${ }^{14}$, qui transforme le banquet en un espace scénique, une mise en scène dont le contenu importe peu : «On ne comprit que la moitié du speech par lequel le président Stéphane Mallarmé ouvrit le banquet; quant à Moréas, on n'entendit rien de ce qu'il dit à cause de l'émotion qui étrangla sa voix ; mais on applaudit avec fureur le toast porté par l'un des assistants qui, gravement, leva son verre et dit: “Je bois à ceux qui ne mangent pas." C'était parfaitement ridicule, et cela a été un parfait succès ${ }^{15}$. »

Ainsi, malgré l'aspect superficiel de réunion d'initiés qu'il peut présenter de prime abord, le banquet en l'honneur de Jean Moréas devient un grand moment d'autoglorification du mouvement symboliste qui prend, par cette mise en scène, une forme visible pour les contemporains, et accède ainsi au statut de groupe en soi dans le champ littéraire de l'époque. Car la littérature est essentiellement fondée par ses représentations : c'est un espace fictionnel, une institution, un rituel qui n'a d'existence que spirituelle. Les banquets littéraires peuvent alors être étudiés comme des cristallisations provisoires de l'espace littéraire, cristallisations qui fonctionnent aussi bien sur un plan social que sur un plan imaginaire.

\section{Scénographie narrative et illustrée des banquets.}

Léon Deschamps est pleinement conscient de la fonction de représentation de ces réunions lorsqu'il décide d'instituer sa série de banquets. En plus d'être des événements de sociabilité, les banquets de la Plume sont destinés à être mis en récit et publiés, formant des vitrines de l'état de la littérature contemporaine.

Un poète comme Mallarmé, qui présida par deux fois les Dîners de la Plume, avait lui aussi une conscience très nette de la théâtralité de ces réunions. Lorsqu'on lui demande de remplacer Leconte de Lisle qui devait présider le banquet du 6 décembre 1892, il signale précisément à Deschamps la fonction qu'il souhaite s'attribuer lors de ce dîner :

\footnotetext{
${ }^{14}$ W. G. C. Byvanck, Un Hollandais à Paris en 1891 : Sensations de littérature et d'art, Perrin, 1892, p. 76-77.

15 Ibidem.
} 
Leconte de Lisle m'a dimanche, au Luxembourg, exprimé l'affectueux désir, que j'occupasse, à votre prochain dîner, la place qui lui appartient selon vous, tous et moi. Je ne saurais ne pas y déférer ; mais j'aime que ce soit ainsi que vous l'entendez avec un tact précis, non pas comme président ; simplement le lecteur, par lui désigné, de la Lettre, à laquelle vous faites allusion. Tout garde ainsi sa perspective ${ }^{16}$.

Ce rôle de lecteur effacé rappelle la vision de l'auteur selon Mallarmé, et son «Toast» prononcé lors de son propre banquet ira dans le même sens. Mais Mallarmé se plie également au protocole des dîners, avec leurs tables d'honneur, leurs hiérarchisations des convives, leurs chronologies. Cette scénographie trouve naturellement sa place dans les comptes rendus des banquets, soigneusement rédigés pour indiquer les positions symboliques de chacun dans ces événements hautement ritualisés.

Les premiers comptes rendus de ces manifestations sont rejetés sur les couvertures de La Plume, qui affichent avis et publicités divers, et disparaissent souvent lorsque les cahiers sont assemblés en recueil ${ }^{17}$. À partir du cinquième Dîner, ces comptes rendus trouvent cependant droit de cité dans le corps même de la revue : de simples réunions amicales, les banquets de la Plume sont devenus des événements littéraires à part entière, qui participent à la configuration du monde des Lettres de leur époque. La relation anonyme du «Cinquième banquet de LA PLUME» dans le numéro du 15 novembre 1892 de la revue affiche clairement les ambitions symboliques de ces manifestations :

Mardi, 8 Novembre 1892. À la table d'honneur: Auguste Vacquerie, président, ayant à sa droite Émile Zola et François Coppée, à sa gauche, Jules Claretie et Aurélien Scholl ; en face du président, Léon Deschamps, ayant à sa droite Y. Rambosson (le plus jeune des poètes présents) et Marcel Bailliot, à sa gauche Raymond Lotthé (le plus jeune des artistes-peintres) et Léon Dequillebecq. Un peu partout, le reste des cent convives ${ }^{18}[\ldots]$.

Sans parler de l'accent mis sur le nombre important de dîneurs, on peut noter la précision apportée à la description de la table d'honneur. En face du président de la soirée, entouré des quatre présidents des dîners précédents qui forment une sorte de panthéon de l'ancienne génération, on trouve Léon Deschamps, flanqué de deux représentants de la littérature et des arts choisis essentiellement pour leur âge. Le directeur de La Plume apparaît bien dans une fonction de passeur générationnel, la scénographie du banquet matérialisant sa fonction sociale.

\footnotetext{
${ }^{16}$ Stéphane Mallarmé, Lettre à Léon Deschamps, Paris, 30 novembre 1892, MCCCLXVIII, Correspondance, $\mathrm{t}$. V, Gallimard, 1981, p. 160.

${ }^{17}$ Il m'a ainsi été impossible, malgré mes recherches, de trouver un exemplaire de La Plume complet de sa couverture pour le quatrième Banquet présidé par Jules Claretie en juin 1892.

18 «Le Cinquième banquet de LA PLUME », La Plume, n 86, 15 novembre 1892, p. 496.
} 
Les comptes rendus suivent un modèle canonique, dressant, après la description de la table d'honneur, la liste des principaux invités, avant de retranscrire quelques lettres amicales d'écrivains absents lues pendant la soirée - surtout si ces lettres permettent d'affirmer que ces banquets portent leurs fruits ${ }^{19}$. Les soirées s'achèvent avec l'élection du président du banquet suivant, saluée par une ovation des convives.

Ces comptes rendus apparaissent également dans la presse quotidienne et dans les revues amies, contribuant à diffuser l'image d'une avant-garde littéraire soucieuse de ménager ses aînés et unie dans un même amour de la beauté. Léon Deschamps apprécie particulièrement cette publicité faite à ses Banquets; il a soin d'annoncer à Mallarmé le succès de la soirée du 6 décembre par son retentissement dans la presse :

Articles sur le banquet, dans le Gil Blas, le Journal, l'Écho de Paris, l'Événement, le Parti National, La Cocarde, Le Gaulois, le Voltaire, le Parisien, le Courrier du Soir, le Paris, le National, la Petite République, le Pays, le Patriote, le Rappel, le Radical, La Libre Parole, la Presse, la Petite Revue, Le Nouveau Siècle, l'Éclair, la Marseillaise, etc. etc ${ }^{20}$.

La présence des journalistes aux tables des banquets est également soigneusement notée dans les comptes rendus de la Plume ${ }^{21}$. Le choix d'offrir à Francis Magnard, directeur du Figaro, la présidence du neuvième banquet, n'est évidemment pas anodin, et permet dès le lendemain la parution d'un article élogieux sur La Plume dans le quotidien, qui rendra compte régulièrement des banquets suivants ${ }^{22}$. Même les comptes rendus les plus critiques de l'entreprise rappellent le but fraternel des banquets, comme cet écho des Entretiens politiques et littéraires de juin 1892, à la suite du Banquet Coppée du 10 mai :

Une revue parisienne, La Plume, a trouvé le moyen qu'on cherchait, hier encore, de faire défiler avec grâce le naturo-parnassisme : ces messieurs se sont montrés, jusqu'ici (11 mai) tels, devant leur jeune auditoire, qu'on ne les soupçonnerait pas d'avoir, dans l'huis-clos de l'Interview, traité les "Symbolistes" qui de "fumistes", qui “d'empoisonneurs". Ne leur

\footnotetext{
${ }^{19}$ Voir par exemple la lettre de Brunetière dans le compte rendu anonyme du « $10^{\text {eme }}$ Dîner de "La Plume" ", $L a$ Plume, $\mathrm{n}^{\circ} 109,1^{\text {er }}$ novembre $1893, \mathrm{p} ; 467$.

${ }^{20}$ Léon Deschamps, Lettre à Stéphane Mallarmé, Paris, 9 décembre 1892, dans Stéphane Mallarmé, Correspondance, t. V, Gallimard, 1981, p. 169.

${ }^{21}$ Voir par exemple le compte rendu du «Sixième Banquet de LA PLUME », La Plume, $\mathrm{n}^{\circ}$ 88, 15 décembre 1892, p. 532 : «A A la table de la presse quotidienne : MM. Georges d'Esparbès, Paul Brulat, Auguste Marin, du Journal, Jean Carrère et Jehan Soudan, du Figaro, Édouard d'Arbourg, du Gil Blas, Arthur Bernède, du Pays, Léon Durocher et Carolus Tenib, du Fin de Siècle, Leroux (représentant le Nain Jaune) de l'Écho de Paris, Gustave Piel, du Rappel, J.-L. Croze, de l'Événement, Jacques des Gachons, du Voltaire et du Télégraphe, Émile Goudeau, de La France, Olivier de Gourcuff, de l'Étendard, et Ed. Bourgès, représentant de l'Agence Dalziel, dont le directeur est notre éminent confère Harry Allis. »

${ }^{22}$ Voir le Figaro du 12 mai 1892. Le Banquet Heredia est mentionné dans les échos du journal le 15 octobre 1893, le Banquet Reyer dans l'édition du 8 avril 1894. Il n'est pas fait mention du Banquet Rodin du 9 décembre 1893, la bombe d'Auguste Vaillant à la Chambre des députés le même soir occupant toute l'actualité, ni du Banquet Puvis de Chavannes du 10 février 1894.
} 
tenons donc compte, généreusement, que de leurs toasts mielleux, et félicitons ceux qui prirent l'initiative de cet apprivoisement tempestif ${ }^{23}$.

Les banquets de la Plume, en plus des fonctions sociales traditionnelles de ce genre de manifestation, prennent ainsi une ampleur nouvelle par l'invitation massive de la presse. La représentation de la gent littéraire en est démultipliée ; par l'utilisation des organes des mass media, ils produisent précisément ce que cette nouvelle donne de l'information déniait: le sentiment d'une relation directe entre le public et les auteurs, l'ouverture du cénacle à la foule $^{24}$. Ces rituels de la sociabilité littéraire, destinés au départ à pallier l'isolement grandissant des écrivains en instituant des cercles communautaires au sein desquels une communication directe, de type aristocratique, était possible, deviennent ainsi les premières manifestations d'une représentation de masse des écrivains au sein de la presse, représentation qui influe considérablement sur l'image même de la littérature, et donc sur les manières de lire des contemporains : on n'interprète pas de la même manière une œuvre de Mallarmé selon qu'il est présenté dans la presse comme un malade mental, ou un égal de Zola.

Les discours des présidents, lus traditionnellement au dessert et publiés en première page de la revue dans la livraison qui suit le banquet, occupent aussi une place importante dans la fonction de représentation de ce monde littéraire ${ }^{25}$. Aurélien Scholl, Émile Zola, François Coppée, Jules Claretie se plient tous au jeu, buvant à la Jeunesse destinée à les dépasser. Ainsi de Zola le 12 avril 1892 :

Messieurs, je bois à la jeunesse, qui est la grande force et le grand espoir, je bois à l'élargissement, au renouvellement de toutes les formules, je bois à l'art de demain qui forcément ne sera plus celui que nous avons apporté, je bois à tout ce que vous allez créer de nouveau, je bois même, si vous le voulez, à l'enterrement des aînés, mais je vous demande de leur faire au moins de belles funérailles ${ }^{26}$.

Ainsi de Coppée ( $1^{\text {er }}$ juin 1892), choisissant lui aussi la forme de la boutade :

Jeunes cadets, merci d'être bon pour un vieux !

[...] Je n'ai point vieilli trop, puisque j'admire encore

Les vers que le soleil des vingt ans fait éclore...

Je bois à la Jeunesse ; elle a toujours raison ${ }^{27}$ !

\footnotetext{
${ }^{23}$ Anonyme, « Notes et notules », Entretiens politiques et littéraires, n 27 , juin 1892, p. 274.

${ }^{24}$ Voir Alain Vaillant, La Crise de la littérature: Romantisme et modernité, Grenoble, ELLUG, coll. Bibliothèque stendhalienne et romantique, 2005.

${ }^{25}$ Les discours d'Émile Zola, de François Coppée et de Jules Claretie apparaissent également dans le livre de Léon Maillard, La Lutte idéale : Les Soirs de La Plume, Sevin / La Plume, 1892.

${ }^{26}$ Émie Zola, discours sans titre, La Plume, $\mathrm{n}^{\circ} 73,1^{\mathrm{er}}$ mai 1892, p. 187.

${ }^{27}$ François Coppée, «Sonnet », La Plume, n $^{\circ} 75,1^{\text {er }}$ juin 1892, p. 239.
} 
Le sonnet de Mallarmé connu sous le titre de «Salut», qui ouvre ses Poésies dans d'édition Deman, doit également être lu dans la série des «Toasts » portés par les présidents des Dîners de la Plume — c'est d'ailleurs sous le titre de «Toast » qu'il paraît en première page de la revue le 15 février 1893 :

Nous naviguons, ô mes divers

Amis, moi déjà sur la poupe

Vous l'avant fastueux qui coupe

Le flot de foudres et d'hivers ${ }^{28}$

On retrouve ici une variation sur le modèle imposé des relations entre la jeunesse et la vieillesse, avec une polarisation du monde littéraire entre deux générations représentées ici par la poupe et la proue. En reprenant ces vers dans son recueil, en choisissant de faire de ce sonnet l'incipit de ses œuvres poétiques, Mallarmé en modifie la portée, mais reconnaît aussi l'importance de cette mise en scène de l'espace littéraire, indispensable à la création de l'œuvre et à sa réception.

Les invitations participent également à la mythologie de ces banquets. Insérées dans les pages de la revue, elles sont illustrées par les collaborateurs habituels de la Plume, qui puisent leur inspiration dans les œuvres des présidents ou dans l'imagerie du symbolisme. L'invitation du troisième dîner, sous la présidence de François Coppée (10 mai 1892), illustrée par Andhré des Gachons, représente ainsi un banquet médiéval animé par un ménestrel devant le Café du Soleil d'or où avaient lieu les soirées littéraires et musicales ${ }^{29}$ [Figure 2]. Ces invitations peuvent également prendre la forme du portrait charge, comme celle du Banquet Vacquerie (8 novembre 1892), où Léon Lebegue le met en scène accompagné de ses quatre vice-présidents qui battent le rappel autour d'une allégorie de la Plume assise sur une table de banquet ${ }^{30}$ [Figure 3]. Mais ce sont les représentations symboliques de l'espace littéraire qui apparaissent dans ces illustrations qui se révèlent les plus intéressantes. Fernand Fau dessine ainsi pour le Banquet Leconte de Lisle (6 décembre 1892) une jeune cuisinière, une plume fichée sur la toque, distribuant le souper à des convives affamés qui lui tendent leurs assiettes [Figure 4]. On reconnaît au premier plan Verlaine (un habitué des banquets, qui réclamait sans cesse dans ses lettres à Deschamps la date des prochaines réunions, et présidera le huitième Dîner ${ }^{31}$ ), qui semble inviter de la main d'autres convives à rejoindre la troupe, puis les cinq vice-présidents et, au fond, un défilé de

\footnotetext{
${ }^{28}$ Stéphane Mallarmé, « Toast », La Plume, n 92, 15 février 1893, p. 67.

${ }^{29}$ Reproduit dans La Plume, $\mathrm{n}^{\circ} 73,1^{\text {er }}$ mai 1892, p. 212.

${ }^{30}$ Reproduit dans La Plume, $\mathrm{n}^{\circ} 85,1^{\mathrm{er}}$ novembre 1892, p. 478.

${ }^{31}$ Voir Paul Verlaine, Lettres inédites à divers correspondants, publiées et annotées par Georges Zayed, Genève, Droz, 1976, p. 127-128.
} 
littérateurs menés par Stéphane Mallarmé - qui se trouvera justement être le président du banquet suivant ${ }^{32}$. Derrière le comique de la situation dépeinte, la hiérarchie des présidents est respectée, et la Plume, sous une forme allégorique, dispense ses bienfaits aux littérateurs qui l'entourent. Mais c'est l'invitation au septième banquet, présidé par Stéphane Mallarmé, qui incarne le mieux la symbolique de ces réunions ${ }^{33}$ [Figure 5]. Léon Deschamps, entouré des rédacteurs de la revue, invite d'un geste large le destinataire de l'invitation à rejoindre la foule d'écrivains qui fait la queue derrière lui pour escalader une gigantesque plume, dont la pointe mène à une tribune solaire surélevée où les six anciens présidents accueillent Stéphane Mallarmé, perché au sommet de la plume et suivi de Verlaine. Cette tribune décorée de palmes et d'une lyre représente bien le Panthéon imaginaire auquel accèdent les présidents des Dîners de la Plume.

\section{Modèles.}

Pour bien comprendre la portée de l'entreprise de Deschamps, il faut également en dégager les modèles implicites. Les Dîners de la Plume ne modifient efficacement l'espace littéraire que dans la mesure où participants et public peuvent découvrir une signification symbolique derrière ces rituels sinon banals.

Le premier modèle important de ces réunions est celui des banquets républicains. Les Dîners de la Plume reproduisent en effet en partie, au sein de la République des Lettres, les valeurs de la Troisième République, telles qu'elles pouvaient se matérialiser par le rituel des banquets : liberté d'expression, neutralité, respect des tendances adverses, les tensions devant se résoudre de manière pacifique dans un espace littéraire œcuménique. Les Banquets de la Plume contribuent à la formation d'un Panthéon de Grands Hommes qui forme un canon dans lequel les jeunes générations peuvent puiser leurs modèles — Léon Deschamps parle dans une lettre à Stéphane Mallarmé d'une « académie idéale ${ }^{34}$ ». Une forme de filiation imaginaire se met ainsi en place, les artistes formant une communauté unie symboliquement par la table du dîner, dont la disposition figure le passage de relais des générations. Le phénomène de l'élection collégiale des présidents des Banquets, désignés par leurs prédécesseurs, assure également l'unité d'un groupe qui pose ses propres frontières et trouve en lui-même sa légitimité. Ce modèle républicain est cependant contrebalancé par l'élitisme affirmé de la manifestation, qui n'accueille que des invités choisis. Le terme de Dîner choisi au départ va

\footnotetext{
${ }^{32}$ Reproduit dans La Plume, $\mathrm{n}^{\circ} 87,1^{\text {er }}$ décembre 1892, p. 518.

${ }^{33}$ Invitation illustrée par Butel et Valton, reproduite dans La Plume, n 91, $1^{\text {er }}$ février 1893, p. 66.

34 Léon Deschamps, Lettre à Stéphane Mallarmé, Paris, 23 janvier 1893, dans Stéphane Mallarmé, Correspondance, t. VI, Gallimard, 1981, p. 36.
} 
d'ailleurs dans le sens d'un certain snobisme : la République des Lettres s'affirme comme une aristocratie ${ }^{35}$.

Un autre modèle apparaît dans la correspondance de Léon Deschamps, celui des douze dieux de l'Olympe. Au départ, c'est simplement le caractère mensuel de l'événement qui pousse Deschamps à n'organiser que douze banquets, comme le rapporte Aurélien Scholl, le premier président des Dîners, dans le numéro spécial de la Plume consacré à son directeur, brusquement emporté par la maladie le 28 décembre 1899, alors qu'il venait de lancer une nouvelle série de banquets :

[...] si cette première soirée réussit, que comptez-vous faire ?

- Continuer, simplement. Douze banquets, un par mois. Suspension d'une année ou deux, puis reprise des réunions pour consacrer les nouveaux, les adeptes, ceux qui seront entrés dans les rangs ${ }^{36}$.

Mais ce chiffre de douze prend rapidement un sens symbolique ; Mallarmé en a conscience lorsqu'il loue le choix de Deschamps de «limit[er] à 12 le chiffre des élus ${ }^{37}$ »: «Votre intention de limiter notre Olympe à douze est d'une haute sagesse ${ }^{38} »$. La première série des banquets comprendra pourtant treize dîners ${ }^{39}$ - conséquence d'une modification du projet initial sous l'impulsion de Coppée pour intégrer les Beaux-arts au Panthéon :

— «Je crois qu'il serait bon de ne pas clore la série des Dîners sans avoir fait à l'artiste qui répond le plus à votre idéal, cela dans chaque branche de l'art, peinture, sculpture, musique, etc., le même honneur que celui fait aux hommes de lettres présents ici... »

Ainsi parlait M. Coppée, à l'un des derniers dîners ; MM. Zola, Scholl et Vacquerie approuvèrent ${ }^{40}$.

Le sculpteur Rodin (le 9 décembre 1893), le peintre Puvis de Chavannes (le 10 février 1894) et le musicien Ernest Reyer (le 7 avril 1894) rejoignent ainsi tour à tour la liste des présidents des Dîners de la Plume. Le compte rendu du dernier banquet est rédigé par Léon Deschamps lui-même, qui affirme avec satisfaction avoir atteint son but :

C'était le dernier et jamais peut-être n'avait régné semblable entrain. [...] Maintenant l'Olympe est fermé. Nous avons fêté nos aînés des Lettres, de la Peinture, de la Sculpture et de

\footnotetext{
${ }^{35}$ Voir le numéro 124 de La Plume, daté du 15 juin 1894, consacré à l'aristocratie.

${ }^{36}$ Aurélien Scholl, «Léon Deschamps, Fondateur de La Plume », La Plume, n 260, 15 février 1900, p. 67.

37 Léon Deschamps, Lettre à Stéphane Mallarmé, Paris, 23 janvier 1893, dans Stéphane Mallarmé, Correspondance, t. VI, Gallimard, 1981, p. 36.

${ }^{38}$ Stéphane Mallarmé, Lettre à Léon Deschamps, Paris, 24 janvier 1893, MCDXI, Correspondance, t. VI, Gallimard, 1981, p. 37.

${ }^{39}$ L'invitation de Banquet Reyer portant par erreur le numéro XIV, plusieurs commentateurs ont cru que la première série des Dîners de la Plume comportaient quatorze banquet; mais le menu porte bien le numéro XIII. Voir la reproduction de ces pièces dans La Plume, $\mathrm{n}^{\circ}$ 120, 15 avril 1894, p. 151 et 152.

${ }^{40}$ Anonyme, « Onzième Banquet de La Plume », La Plume, n 112, 15 décembre1893, p. 540.
} 
la Musique comme il convenait et ces réunions amicales ont eu le résultat souhaité : la fraternité remise en honneur parmi tous les membres de notre grande famille artiste ${ }^{41}$.

Le dernier grand modèle de ce type de réunion est évidemment la Cène, comme le note ironiquement Camille Mauclair dans ses souvenirs de la vie littéraire :

Le fait de manger en commun pour honorer la pensée d'un homme ne saurait plus être discuté dans son efficacité morale depuis la Cène, et c'est là une des formes essentielles du répertoire honorifique de l'humanité. J'ai donc tout à fait tort de considérer les banquets littéraires et artistiques comme des corvées glaçantes ou de déplaisantes bamboches, mais je mentirais en les définissant autrement, car ils m'apparurent toujours tels ${ }^{42}$.

Le banquet est une forme de manifestation religieuse ; Fontainas rappelle ainsi que le banquet en l'honneur du Pèlerin passionné s'était transformé en une vaste «manifestation de solidarité : «Dans ce culte du Beau désintéressé, communiaient, sans parti-pris d'école et sans embrigadement, les esprits les plus divers, tous les esprits sensibles, émus de poésie ${ }^{43} »$. Il s'agit, une fois de plus, d'affirmer la cohésion d'une communauté par une réunion à valeur commémorative.

La réalité des banquets de la Plume ne correspond malheureusement pas toujours avec ces modèles idéaux, comme on peut le constater à la lecture des journaux intimes et des mémoires des témoins de l'époque. Si Retté note que même les plus intimidés des présidents finissent par se rassurer devant la jeunesse littéraire, la crainte d'un mauvais coup les laisse toujours sur leurs gardes. José-Maria de Heredia peine à se départir d'une certaine crainte lors du banquet qu'il préside le 14 octobre 1893: «Nous avions tellement houspillé les Parnassiens que, quoique assuré de notre admiration foncière pour ses beaux sonnets, il semblait craindre qu'on ne réclamât sa tête au dessert, après une invocation au vers libre ${ }^{44}$. » Léon Deschamps apprend à Mallarmé que Leconte de Lisle, qui devait présider le sixième Banquet, préfère se faire porter pâle plutôt que d'affronter une foule de jeunes littérateurs :

Vous savez que M.M. Scholl, Zola, Claretie, Coppée et Vacquerie l'avaient désigné pour présider notre sixième banquet. Or, le cher poète a peur de se trouver avec Verlaine et Moréas et il invoque son état de santé, malgré les supplications de Vacquerie et les nôtres, pour ne pas assister au banquet ${ }^{45}$.

Jules Renard rapporte dans son Journal des scènes de laideur et de saleté. Le 9 mars, au lendemain du premier banquet, il n'est pas tendre pour ses condisciples :

\footnotetext{
${ }^{41}$ Léon Deschamps, « La Quinzaine artistique et littéraire », La Plume, n 120, 15 avril 1894, p. 150-151.

${ }^{42}$ Camille Mauclair, Servitude et Grandeur littéraires, Ollendorff, 1922, p. 96.

${ }^{43}$ André Fontainas, Mes Souvenirs du Symbolisme, op.cit., p. 77.

${ }^{44}$ Adolphe Retté, Le Symbolisme : Anecdotes et Souvenirs, Vanier, 1903, p. 175.

${ }^{45}$ Léon Deschamps, Lettre à Stéphane Mallarmé, Paris, 29 novembre 1892, dans Stéphane Mallarmé, Correspondance, t. V, Gallimard, 1981, p. 159.
} 
Hier, dîner de La Plume. Rares, les figures intelligentes d'hommes intelligents. Des laideurs étudiées comme des têtes de cannes.

[...] Et ce dîner. Les mains noires du garçon, les choses noires des assiettes, ce gigot laineux mangé dans des soucoupes... Willy va faire des provisions chez le charcutier voisin. C'est drôle ! Seulement, il ne les mange pas, et les saucissons brandis ne sont que prétextes à clameurs...

[...] La morale du dîner, c'est que le restaurateur s'est aperçu qu'au moins seize d'entre nous n'avaient pas payé.

- C'est beau, le génie ! dit Dubus. Ça donne le droit d'être un cochon, et d'imposer ses vices et ses poux. On trouve ça naturel.

$\mathrm{Ah}$ ! parmi nos petites gloires lumineuses, que de bouts de chandelles ${ }^{46}$ !

Edmond de Goncourt rapporte également le 12 février 1893 le récit du Banquet Mallarmé par Rodenbach, lors duquel Verlaine s'était ridiculisé par ses excès de boisson et ses balbutiements sadiques ${ }^{47}$.

\section{La construction d'un espace littéraire.}

Malgré ces faux pas, les Dîners de la Plume permettent la reconfiguration de l'espace littéraire sur deux niveaux complémentaires, social et imaginaire. Le champ littéraire de la fin du XIX ${ }^{\mathrm{e}}$ siècle, tel qu'il est décrit par Bourdieu, est divisé selon une opposition jeunes / aînés (les premiers jouissant d'un bas degré de consécration contrairement aux seconds), et selon une opposition entre la «production pure », destinée au marché restreint des littérateurs (les petites revues pour les jeunes, le Parnasse pour les aînés), et la «grande production », orientée vers le grand public (le Cabaret pour les jeunes, le Théâtre de boulevard pour les aînés), Zola se situant dans un entre-deux difficile ${ }^{48}$. Or les listes des présidents et des convives des banquets de la Plume tendent à contredire ces analyses, en mettant en place un espace œcuménique dans lequel ces clivages ne fonctionnent pas. Il faut rappeler que l'on trouve parmi les présidents des banquets deux présidents de la Société des Gens de Lettres, Jules Claretie (qui a exercé cette fonction entre 1885 et 1888) et Émile Zola (président de 1891 à 1894, puis après une interruption de 1894 à 1896). Académiciens, dramaturges, journalistes, humoristes, poètes parnassiens, décadents ou symbolistes se réunissent pour célébrer leur communauté d'esprit. Les soirées littéraires et musicales qui suivent les banquets, où se

\footnotetext{
${ }^{46}$ Jules Renard, Journal, Robert Laffont, coll. Bouquins, 1990, p. 96-97.

${ }^{47}$ Edmond et Jules de Goncourt, Journal, Mémoires de la vie littéraire, 1891-1896, avant-propos de l'Académie Goncourt, texte intégral établi et annoté par Robert Ricatte, t. IV, Fasquelle / Flammarion, 1956, p. 364-365.

${ }^{48}$ Voir Pierre Bourdieu, Les règles de l'art: Genèse et construction du champ littéraire (1992), Éditions du Seuil, coll. Points Essais, 1998, p. 204-205.
} 
succèdent poètes et chansonniers, remettent en cause la hiérarchie des genres et l'opposition entre une littérature d'élite et une littérature de masse. S'il y a un "ennemi », ici, c'est le théâtre de boulevard, représenté par la figure ridiculisée de Francisque Sarcey ; au-delà des différences d'âge ou d'esthétique, c'est l'opposition à une littérature mercantile considérée comme non artistique qui unit les convives des Dîners de la Plume.

Ces manifestations ne se contentent pas de consolider les liens sociaux entre les gens de Lettres. Les conditions de la réception des textes sont en partie définies par les groupes qui lisent et par les canons institués ; redéfinir ces éléments, c'est donc redéfinir la façon de lire, redéfinir la littérature et le sens même des textes - non seulement pour les acteurs directs que sont les invités, mais également pour les spectateurs indirects que sont les lecteurs de journaux diffusant des comptes rendus de ces réunions. Des institutions aussi anodines que ces banquets participent donc activement à la configuration de l'espace littéraire. De nouveaux canons sont mis en place, des relations virtuelles entre des œuvres sont découvertes : à partir du moment où le nom d'un auteur, sa notoriété, sa représentation dans la presse, deviennent des caractéristiques fondamentales de la réception de ses œuvres, la position sociale d'un écrivain peut déterminer un repositionnement de la valeur littéraire de son œuvre dans la bibliothèque personnelle de chacun. Les banquets reproduisent ainsi, dans l'espace réel des salles qui accueillent ces manifestations, l'agrégation des fidèles autour d'un maître, la soumission des œuvres des disciples à celles des grands auteurs. Les « institutions de la vie littéraire » (académies, groupes, réunions) et les «institutions littéraires » (genres, formes, théories ${ }^{49}$ ) ne sont pas séparées de façon stricte : leurs frontières sont poreuses, et le social et l'imaginaire s'informent, se consolident, s'influencent réciproquement.

En proposant de nouveaux canons, les banquets de Deschamps redéfinissent les groupes littéraires, et créent de nouvelles façons d'appréhender les œuvres, ce qui n'est pas toujours pour plaire à certaines communautés de lecteurs qui peuvent refuser de se reconnaitre dans ces nouvelles normes littéraires. C'est le cas d'Yvanhoé Rambosson, jeune critique symboliste qui refuse de faire coïncider son propre canon littéraire avec celui d'Émile Zola, tout en reconnaissant sa valeur dans le monde littéraire :

Vous avez fait - deux fois déjà - monsieur, aux jeunes hommes soucieux d'art nouveau, l'honneur d'assister au banquet de La Plume. Ce fut loyal, de vous, un maître si vous

\footnotetext{
${ }^{49}$ Voir Alain Viala, Naissance de l'écrivain : Sociologie de la littérature à l'âge classique, Paris, Minuit, coll. Le Sens commun, 1985.
} 
n'êtes pas le nôtre, de vous, glorieux de labeurs accomplis, envers nous, plus riches de projets et d'espérances que d'histoire. Nous vous en remercions ${ }^{50}$.

Car les maîtres que reconnaît Rambosson, ce sont Verlaine, Mallarmé, Nerval, Villiers ; s'il inclut également Balzac dans son Panthéon, c'est en prenant soin de préciser de quel Balzac il s'agit :

Il reste Balzac dont vous vous réclamez, mais qui n'est point des vôtres. Réaliste c'est incontestable, il le fut souvent; cependant il ne fut point que cela et bien plutôt son œuvre se rapproche de ce type qui n'est point pour nous déplaire d'une œuvre idéaliste partant d'une profonde connaissance et d'un profond sentiment de la vie ${ }^{51}$.

L'Olympe des Banquets de la Plume n'est lui-même qu'une proposition de définition de l'espace littéraire, une représentation parmi d'autre qui tente de s'imposer. La plus grande difficulté rencontrée par Léon Deschamps dans la création de cet espace œcuménique qui est aussi un espace imaginaire reste cependant la manière toute aléatoire dont ce canon se forme, les présidents étant élus aussi bien en mesure de l'admiration que leur porte leurs prédécesseurs que selon les bienséances sociales qui impliquent que certains littérateurs en vue se voient proposer de siéger en qualité de président. C'est ce que Léon Deschamps tente de faire comprendre avec tact à Mallarmé lorsqu'il lui demande un nom pour son successeur :

Vous êtes seul maître quant au choix. Ces jours derniers, j'ai reçu la visite de Descaves qui, sans en avoir l'air, devait venir tâter le terrain pour son protecteur E. de Goncourt, - car l'auteur de Sous-Offs m'a parlé de cela et m'a dit que M. de Goncourt est peiné par notre attitude vis à vis d'A. Daudet, que si «on le désignait» pour un banquet, il serait gêné... D'autre part, l'ensemble des suffrages ou plutôt des prévisions de nos camarades indiquent différents noms : Verlaine (et l'on tremble que le jour du dîner notre ami soit gris comme à son ordinaire), Huysmans, Becque (ce dernier semble favori), Richepin et de Goncourt.

Tout cela n'est qu'un renseignement donné par moi pour vous indiquer les préoccupations $^{52}$.

Mallarmé impose cependant Verlaine [Figure 6] ; Edmond de Goncourt refusera de toute manière la présidence en mai 1893, malgré l'insistance de Deschamps et de Francis Magnard, par solidarité avec Daudet que certains écrivains attaquaient avec virulence dans la Plume $^{53}$. En refusant de présider un banquet de Deschamps, Edmond de Goncourt faisait plus

\footnotetext{
${ }^{50}$ Yvanhoé Rambosson, « À Monsieur Émile Zola », La Plume, n 75, $1^{\mathrm{er}}$ juin 1892, p. 240.

${ }^{51}$ Ibidem.

52 Léon Deschamps, Lettre à Stéphane Mallarmé, Paris, 23 janvier 1893, dans Stéphane Mallarmé, Correspondance, t. VI, Gallimard, 1981, p. 36.

${ }^{53}$ Voir Edmond et Jules de Goncourt, Journal, op. cit., t. IV, p. 398-399.
} 
que de s'isoler du reste de la communauté littéraire : il engageait aussi un certain point de vue sur la littérature, et contribuait par la négative à la configuration du champ littéraire.

Peu avant sa mort, Léon Deschamps entame une nouvelle série de Banquets autour d'auteurs de sa génération avec Paul Adam (le 7 décembre 1899), qui devait être suivi de Jean Moréas le 11 janvier 1900. Dans le compte rendu du banquet préparatoire au Dîner de Paul Adam, Deschamps fait le bilan de la première série des Banquets : «le résultat fut que dans la grande famille des lettres et des arts, on se respecta mutuellement et que la place de chacun dans la hiérarchie du talent ou du génie fut officiellement reconnue : Zola ne fut plus un “cochon”, Mallarmé, un “fabricant de rébus", Verlaine, un “ivrogne”, Rodin, un "gâcheur de plâtre" à peine digne du titre de maçon, Puvis, un toqué à la manie inoffensive ${ }^{54}$. » La reprise des Banquets est justifiée par une nouvelle division des écrivains causée par l'Affaire de triste mémoire : «Des haines nouvelles sont apparues entre nous : cette fois on ne se bat plus pour la suprématie d'un art, mais l'on se méprise au nom d'une justice qui prend diverses formes suivant la couleur confessionnelle de 1'éducation reçue ${ }^{55}$.» Le titre de président disparaît pour mieux marquer le caractère convivial de ces manifestations, qui ne visent pas à asseoir l'influence littéraire d'un auteur en particulier.

La mort de Deschamps met fin à cet idéal. Les banquets qui suivront, sous la direction de Karl Boès, ne visent plus qu'à être des réunions amicales, pour «la joie d'être ensemble $^{56}$ ». L'idéalisme somme toute assez naïf de Deschamps est abandonné; la scénographie devient parodique (« À la table d'honneur — pour employer l'usuel cliché $\left.{ }^{57} »\right)$; il ne s'agit plus d'aider la jeunesse à s'épanouir, mais de proposer un idéal moral $^{58}$. Le retentissement dans la presse est presque nul : «Cette même grande presse d'ailleurs, celle qui décrit les toilettes de Longchamp et donne le texte des discours de M. Paul Deschanel aux pompiers d'Eure-et-Loi [sic], a jugé oiseux de rendre compte d'un banquet littéraire que présidait l'auteur d'une pièce en plein succès et de reproduire un toast d'Octave Mirbeau à la

\footnotetext{
${ }^{54}$ Léon Deschamps, «Reprise des Banquets de "La Plume"” ", La Plume, n 245, 15 novembre 1899, p. 748.

55 Idem, p. 749.

${ }^{56}$ Anonyme, «Le XVIe banquet de "La Plume"” (Octave Mirbeau, 6 juin 1903), La Plume, n 340, 15 juin 1903, p. 709.

57 Ibidem.

${ }^{58}$ Idem, p. 710.
} 
jeunesse $^{59}$. » Les banquets s'espacent, deviennent annuels (on fête alors l'anniversaire de la fondation de la revue), puis disparaissent avec la Plume en $1905^{60}$.

\section{Annexe : Liste des Dîners de la Plume.}

I. Aurélien Scholl, 8 mars 1892, Café-Restaurant des Étudiants, 41 rue des Écoles.

II. Émile Zola, 12 avril 1892, même lieu.

III. François Coppée, 10 mai 1892, Café des Lettres et des Arts, 42 rue des Écoles.

IV.Jules Claretie, [ ?] juin 1892, lieu inconnu.

V. Auguste Vacquerie, 8 novembre 1892, Restaurant du Palais, 5 place St-Michel.

VI. Leconte de Lisle (Stéphane Mallarmé), 6 décembre 1892, même lieu.

VII. Stéphane Mallarmé, 9 février 1893, même lieu.

VIII. Paul Verlaine, 13 avril 1893, même lieu.

IX. Francis Magnard, 11 mai 1893, même lieu.

X. José-Maria de Heredia, 14 octobre 1893, même lieu.

XI. Auguste Rodin, 9 décembre 1893, même lieu.

XII. Puvis de Chavannes, 10 février 1894, même lieu.

XIII. Ernest Reyer, 7 avril 1894, même lieu.

XIV. Paul Adam, 7 décembre 1899, Café Voltaire.

XV. Auguste Rodin, 11 juin 1900, même lieu.

XVI. Octave Mirbeau,6 juin 1903, Restaurant du Palais, 5 place St-Michel.

XVII. XV XV $^{\mathrm{e}}$ anniversaire de La Plume, 21 novembre 1903, même lieu.

XVIII. Émile Verhaeren, 23 janvier 1904, Restaurant des Sociétés Savantes, 8 rue Danton.

XIX. Albert Besnard, 15 avril 1905, même lieu.

\section{Table des illustrations.}

Figure 1 : Benoît Lévy, Invitation illustrée du « $1^{\text {er }}$ Dîner », La Plume, $n^{\circ} 69,1^{\mathrm{er}}$ mars 1892, p. 128.

Figure 2 : Andhré des Gachons, Invitation illustrée du «Troisième Dîner », La Plume, $\mathrm{n}^{\circ}$ 73, $1^{\mathrm{er}}$ mai 1892, p. 212.

Figure 3 : Léon Lebegue, Invitation illustrée du « Cinquième Banquet », La Plume, $\mathrm{n}^{\circ} 85,1^{\mathrm{er}}$ novembre 1892, p. 478.

\footnotetext{
${ }^{59}$ Idem, p. 709.

${ }^{60} \mathrm{La}$ renaissance de la revue, avec une équipe entièrement remaniée, entre 1911 et 1914, n'apporta pas de nouveaux banquets littéraires.
} 
Figure 4 : Fernand Fau, Invitation illustrée du « Sixième Banquet », La Plume, ${ }^{\circ}$ 87, $1^{\text {er }}$ décembre 1892, p. 518.

Figure 5 : Butel et Valton, Invitation illustrée du «Septième Banquet », La Plume, $\mathrm{n}^{\circ}$ 91, $1^{\text {er }}$ février 1893, p. 66.

Figure 6 : F. A. Cazals, Invitation illustrée du « $8^{\mathrm{me}}$ Banquet $»$, La Plume, $\mathrm{n}^{\circ}$ 95, $1^{\mathrm{er}}$ avril 1893, p. 166. 